\title{
CARACTERISTICAS SOCIODEMOGRÁFICAS E MORTALIDADE MATERNA EM UM HOSPITAL DE REFERÊNCIA NA CIDADE DE CURITIBA - PARANÁ*
}

Karin Keffler ${ }^{1}$, Silvana Regina Rossi Kissula Souza ${ }^{2}$, Marilene Loewen Wall ${ }^{3}$, Marialda Martins ${ }^{4}$, Suzana Dal Ri Moreira ${ }^{5}$

RESUMO: A mortalidade materna atrai inúmeras discussões e preocupações no Brasil visto que reflete a qualidade da assistência prestada nos serviços de saúde, bem como a operacionalização das políticas públicas voltadas à saúde da mulher. Esta pesquisa teve como objetivo identificar o perfil sociodemográfico e causal da mortalidade materna no período de 2004 a 2007, em um hospital de ensino da cidade de Curitiba, Estado do Paraná. Após análise das fichas hospitalares de investigação confidencial de óbitos e dos pareceres técnicos do Comitê Estadual de Prevenção de Mortalidade Materna encontrou-se um percentual de $80,6 \%$ de óbitos evitáveis, sendo que $45,16 \%$ destes ocorreram devido às causas diretas de óbito materno. Dentre essas causas, as que mais provocaram óbitos foram aquelas relacionadas a doenças do aparelho circulatório, embolias obstétricas e doenças renais. O estudo possibilitou concluir que há deficiência nos registros de informações nos prontuários quanto aos dados sociodemográficos, bem como a necessidade de discutir a melhoria de acesso aos serviços de saúde com vistas a reduzir as taxas de mortalidade materna.

PALAVRAS-CHAVE: Mortalidade materna; Saúde da mulher; Epidemiologia.

\section{SOCIO-DEMOGRAPHIC CHARACTERISTICS AND MATERNAL MORTALITY IN A REFERENCE HOSPITAL IN THE CITY OF CURITIBA - PARANÁ}

\begin{abstract}
Maternal mortality is in the center of many discussions and is of concern in Brazil since it reflects the quality of care in health services, as well as the operationalization of public policies related to women's health. This research aimed to identify the sociodemographic and causal profile of maternal mortality in the period 2004-2007 in a teaching hospital in Curitiba, Parana State. After analysis of the hospital records during confidential investigation of deaths and technical statements by experts of the State Committee for Prevention of Maternal Mortality, it was found a percentage of $80.6 \%$ of avoidable deaths, of which $45.16 \%$ of these were due to direct causes of mother deaths. Among these causes, the ones which caused more deaths were those related to circulatory diseases, obstetric embolism and kidney disease. We concluded that there's some deficiency in the information registries in the medical record regarding socio demographic data, and there's a need to discuss the health care acess improvement in order to decrease the maternal mortality rates.
\end{abstract}

KEYWORDS: Maternal mortality; Women's health; Epidemiology.

\section{CARACTERÍSTICAS SOCIODEMOGRÁFICAS Y MORTALIDAD MATERNA EN UN HOSPITAL DE REFERENCIA EN LA CIUDAD DE CURITIBA - PARANÁ}

\begin{abstract}
RESUMEN: La mortalidad materna atrae innúmeras discusiones y preocupaciones en Brasil ya que refleja la cualidad de la asistencia prestada en los servicios de salud, así como operacionaliza las políticas públicas para la salud de la mujer. Esta investigación tuvo como objetivo identificar el perfil sociodemográfico y causal de la mortalidad materna en periodo de 2004 a 2007, en un hospital de enseñanza de la ciudad de Curitiba, estado de Paraná. Después del análisis de las fichas hospitalares de investigación confidencial de óbitos y de los pareceres técnicos del Comité Estadual de Prevención de Mortalidad Materna, fue encuentrado un percentual de 80,6\% de óbitos evitables, siendo 45,16\% de estes ocurridos por causas directas de óbito materno. Entre esas causas, las que más provocaron óbitos fueron aquellas referentes a enfermedades del aparato circulatorio, embolias obstétricas y enfermedades renales. Nosotros concluimos que existe deficiencia en los registros de informaciones en los prontuarios cuanto a los datos sociodemográficos, así como a la necesidad de discutir la mejoría de acceso a los servicios de salud para disminuir los índices de mortalidad materna PALABRAS CLAVE: Mortalidad materna; Salud de la mujer; Epidemiología.
\end{abstract}

\footnotetext{
*Artigo extraído de Trabalho de Conclusão de Curso apresentado ao Depto. de Enfermagem da Universidade Federal do Paraná-UFPR, 2009. 'Enfermeira.

${ }^{2}$ Enfermeira. Mestre em Engenharia de Produção com ênfase em Ergonomia. Docente do Departamento de Enfermagem da UFPR. Membro do Núcleo de Estudos, Pesquisa e Cuidado Humano de Enfermagem-NEPECHE.

${ }^{3}$ Enfermeira. Doutora em Enfermagem. Docente do Departamento de Enfermagem da UFPR. Membro do NEPECHE.

${ }^{4}$ Enfermeira. Doutoranda em Enfermagem na Universidade Federal de Santa Catarina-UFSC. Professora Assistente do Departamento de Enfermagem da UFPR. Membro do NEPECHE. Membro do Grupo de Pesquisa em Saúde da Mulher e Recém-nascidoGRUPESMUR/UFSC.

${ }^{5}$ Médica do Serviço de Epidemiologia Hospitalar do Hospital de Clínicas da UFPR.
}

Autor correspondente:

Silvana Regina Rossi Kissula Souza

Universidade Federal do Paraná

Rua Pe. Camargo 120 - 800160-240 - Curitiba-PR, Brasil

Recebido: 28/08/09

E-mail: skissula@ufpr.br

Aprovado: 30/07/10 


\section{INTRODUÇÃO}

A mortalidade materna ainda reflete a falta de qualidade da assistência prestada nos serviços de saúde, bem como a insatisfatória operacionalização das políticas públicas voltadas à saúde da mulher, pois é um evento presente com alta incidência no Brasil e atrai inúmeras discussões e preocupações.

Segundo a definição do Manual dos Comitês de Mortalidade Materna do Ministério da Saúde, morte materna é toda aquela

morte que ocorre durante a gestação ou dentro de até 42 dias após o término da mesma, independente da duração, localização, devido a qualquer causa relacionada ou agravada pela gestação, porém, não devida a causas acidentais ou externas ${ }^{(1: 12)}$.

Estima-se que no mundo, todos os anos, mais de 500 mil mulheres morram de complicações gestacionais e do parto. Pelo menos, sete milhões de mulheres que sobrevivem a essas complicações sofrem sérios problemas de saúde e quase 50 milhões sofrem eventos adversos à saúde como consequência do parto. A maioria dessas doenças e complicações ocorre nos países em desenvolvimento ${ }^{(2)}$.

Os padrões de morbimortalidade encontrados revelam diversidade de doenças e diferenças de desenvolvimento regional e de classe social. Assim sendo, faz-se necessário analisar os óbitos ocorridos para melhor conhecer suas causas, bem como seu comportamento nos estados brasileiros, com vistas a permitir melhor avaliação da situação e apontar sugestões para a efetiva redução do número de óbitos de mulheres em idade fértil ${ }^{(3)}$.

As mortes maternas relacionadas às causas obstétricas são classificadas em dois tipos, as causas obstétricas e as obstétricas indiretas. As causas diretas são aquelas resultantes de complicações relacionadas ao ciclo gravídico puerperal, ocorrendo devido a tratamento incorreto, omissão, intervenção, ou resultantes de uma série de eventos. As mortes por causas obstétricas indiretas são resultantes de doenças que existiam antes da gestação ou que se desenvolveram durante esta e que são agravadas pelas alterações fisiológicas próprias da gestação ${ }^{(1)}$.

No Brasil, a mortalidade materna tem sido motivo de preocupação das autoridades de saúde, no âmbito federal, estadual e municipal. Em um grande número de municípios, há Comissões de Estudo e Prevenção de Mortes Maternas, que investigam os casos de morte materna declarados ou suspeitos, sendo que, em muitos deles, são pesquisadas todas as mortes de mulheres em idade fértil ${ }^{(4)}$.

No Estado do Paraná, segundo os dados do Comitê Estadual de Prevenção da Mortalidade Materna (CEPMM), a razão de mortalidade materna (RMM) para os anos de 2004 e 2005 foi, respectivamente, 69 e 65/100.000 nascidos vivos (nv), ou seja, foram consideradas altas, sendo necessário reduzir em quase sete vezes o número de óbitos ${ }^{(5)}$. No ano de 2008 , o coeficiente foi de 56,4/100.000 n.v e dados preliminares de 2009 mostram um coeficiente de 32,4/100.000 nv ${ }^{(6)}$.

No intuito de contribuir para a compreensão deste fenômeno, este estudo buscou caracterizar a mortalidade em um hospital de ensino, referência no atendimento de gestações de alto risco, tendo por objetivo identificar o perfil sociodemográfico e de causalidade da mortalidade materna no período de 2004 a 2007.

\section{METODOLOGIA}

Trata-se de uma pesquisa retrospectiva exploratório-descritiva, para a qual se utilizou fonte secundária de dados. Foi realizada uma análise dos dados, o que consiste na exposição das características de uma determinada população ou de um determinado fenômeno, estabelecendo conexões entre as variáveis obtidas, onde a fonte de dados reside em registros ou documentos de órgãos públicos ou privados de qualquer natureza $^{(7)}$.

O local de estudo e coleta de dados foi o Serviço de Epidemiologia Hospitalar de um hospital de ensino da cidade de Curitiba, no primeiro semestre de 2009 , e optou-se por extrair as informações das fichas confidenciais de investigação hospitalar de morte materna (elaboradas pelo Ministério da Saúde) e do parecer da câmara técnica da Secretaria de Saúde do Estado do Paraná (SESA), ou seja, do Comitê de Mortalidade Materna do Estado. Esses dados foram organizados a partir de um instrumento de coleta de dados elaborado pelas pesquisadoras e posteriormente analisados em planilha simples do programa Microsoft Excel.

Foram analisados 31 óbitos e caracterizados dentro das variáveis epidemiológicas: cor, idade, estado civil, ocupação, renda, escolaridade, número de residentes no domicilio, zona de residência e, através do histórico ginecológico-obstétrico de cada mulher, o número de consultas realizadas durante o pré-natal, comorbidades existentes, gestações anteriores e o número de filhos vivos. 
Para a elaboração desta pesquisa foram observadas as normas éticas estabelecidas pela resolução 196/96 do Conselho Nacional de Saúde, sendo o projeto aprovado pelo Comitê de Ética em Pesquisa do Setor de Ciências da Saúde da Universidade Federal do Paraná sob registro CEP/SD: 676.011.09.02 - CAAE: 0041.0.208.091-09.

\section{RESULTADOS E DISCUSSÃO}

Em relação ao estado civil, identificamos $61 \%$ de mulheres casadas, $36 \%$ solteiras e $3 \%$ separadas. Entre as funções exercidas predominou a atividade no lar (66\%), seguida de: empregada doméstica, cozinheira, zeladora, operadora de caixa, cabeleleira, ambulante, carrinheira, estudante e desempregada.

Do total das gestantes, $80,65 \%$ foram classificadas como de alto risco pelo CEPMM. Segundo a definição do Manual Técnico do Ministério da Saúde, por gestação de alto risco entende-se "aquela na qual a vida ou saúde da mãe e/ou do feto tem maiores chances de ser atingida por complicações que a média das gestações"(8:13).

Em relação à incidência de complicação na gestação, 70,96\% das gestantes apresentaram algum tipo de complicação, 19,35\% não apresentaram nenhuma complicação e, em 9,6\% dos casos, a informação foi ignorada. Em relação às gestações, $96,77 \%$ delas foram gestações únicas e 3,22\% gestação gemelar. Quanto à paridade, $32 \%$ de primigestas e $68 \%$ de multigestas.

Quanto ao tipo de parto, identificamos 64,51\% de cesarianas, apenas $16,12 \%$ de partos normais e $19,35 \%$ dos casos resultaram em abortos e óbito fetal intrauterino. Em relação ao tipo de parto, no ano de 2005 em Curitiba, em um total de 24.520 nascidos vivos, $10.834(44,2 \%)$ foram por partos vaginais e 13.686 $(55,8 \%)$ por partos cirúrgicos, o que aponta para um aumento no número de cesarianas na população em geral $^{(9)}$.

É importante salientar que no Estado do Paraná foram pactuadas Estratégias de Implementação e Operacionalização dos Serviços e, neste pacto, ajustou-se que os índices de cesarianas de cada unidade hospitalar sejam proporcionais e conforme a complexidade de atenção. Ou seja, para os serviços denominados de baixa complexidade a taxa é de $25 \%$ de cesarianas; para os de média complexidade é de $35 \%$ de cesarianas; e para o de alta complexidade, de $45 \%{ }^{(10)}$. Portanto, o índice de cesarianas encontrado neste estudo ficou acima do esperado para um serviço de referência em alta complexidade.

Observou-se que a duração média das gestações foi de 29 semanas, sendo que $82 \%$ atingiram o terceiro trimestre gestacional; $11 \%$ o segundo trimestre e $7 \%$ delas sustentou-se apenas até ao primeiro trimestre. Entre os nascimentos identificamos 41,93\% de prematuros, $29,03 \%$ de recém-nascidos de termo, $22,58 \%$ de natimortos e $6,45 \%$ de pós-termo.

Podemos afirmar que $33 \%$ dos óbitos acometeram gestantes na faixa etária de 20 a 24 anos, e $26 \%$ atingiram mulheres que tinham entre 30 a 34 anos de idade. Quando somamos o número de mulheres na faixa etária de 20 a 29 anos, obtivemos um total de $49 \%$, ou seja, quase metade das mulheres que foram a óbito estavam em idade produtiva. As mulheres entre $35 \mathrm{e}$ 39 anos que foram a óbito totalizaram $19 \%$ do total e as adolescentes (15 a 19 anos) representaram 3\% dos óbitos, sendo o mesmo valor para as mulheres com mais de 40 anos.

Em relação ao município de residência, $40 \%$ das mulheres moravam em Curitiba e as demais (60\%) residiam em municípios da Região Metropolitana de Curitiba e tendo sido encaminhadas ao Serviço de Pronto-Atendimento de Ginecologia e Obstetrícia (PAGO) do referido Hospital, que é referência para atendimento das gestações de alto risco. Os municípios que mais encaminharam pacientes para este serviço foram Piraquara (7\%), Almirante Tamandaré (7\%), Paranaguá (7\%), Araucária (6\%), Colombo (6\%), São José dos Pinhais (6\%), Mandirituba (3\%), Itaperuçu (3\%), Rio Branco do Sul (3\%), Rio Negro (3\%), Campo Largo (3\%), Tijucas do Sul (3\%) e Pinhais (3\%), ressaltando que somente os Municípios de Paranaguá e Rio Negro não fazem parte da Região Metropolitana de Curitiba.

Observou-se neste estudo que o nível de escolaridade predominante (84\%) entre as mulheres que faleceram foi o ensino fundamental incompleto. Apenas uma mulher (3\%) tinha ensino superior completo e, em 13\% dos casos, a informação constou como ignorada. No item renda, um dos indicadores que refletem a condição social na qual a pessoa ou grupo está inserido, foi observada renda média de até três salários mínimos em $75 \%$.

Nas gestações de alto risco é muito importante que as equipes dos serviços de saúde identifiquem, o mais rápido possível, o problema existente e façam um diagnóstico das doenças ligadas à gravidez. Quanto mais cedo se obtiver o diagnóstico, mais eficiente serão o tratamento e os cuidados necessários, evitando ao 
máximo que ocorram danos à mãe e/ou ao feto. Um dos critérios de inclusão para a classificação como gestação de alto risco é a condição socioeconômica, caracterizada por: baixa escolaridade, trabalho que exige demasiado esforço, baixa renda, exposição a agentes físico-químicos nocivos, situação conjugal insegura e conflitos familiares ${ }^{(11)}$.

A variável raça/cor deve ser analisada com especial atenção, pois permite avaliar questões sociais e até mesmo de acesso aos serviços, pois a ocorrência dos óbitos maternos em sua maioria, acontece nas classes sociais mais excluídas, com baixos salários e escolaridade ${ }^{(12)}$.

Em relação às consultas preconizadas pelo Programa Mãe Curitibana ${ }^{(13)}$, da Prefeitura Municipal de Curitiba, toda gestante da área de abrangência da unidade de saúde inscrita no programa deverá ser acompanhada pela equipe de saúde. Neste contexto, as consultas deverão ser mensais até o $7^{\circ}$ mês, quinzenais durante o $8^{\circ}$ mês e semanais durante o $9^{\circ}$ mês até o parto. Um número mínimo de quatro consultas médicas e três consultas de enfermagem são preconizadas como parâmetro de qualidade de assistência ao pré-natal. Ainda conforme o protocolo de assistência ao pré-natal municipal, quando houver suspeita ou diagnóstico de alto risco, a gestante deverá ser avaliada em um serviço de referência de pré-natal de alto risco e, se o risco for confirmado, este serviço determinará a conduta e o local de acompanhamento desta gestante, a qual será vinculada a uma maternidade de alto risco ${ }^{(13)}$.

Neste estudo, o número médio de consultas de pré-natal foi de sete consultas em $74 \%$ das gestantes, sendo que o percentual de mulheres que não realizou consulta de pré-natal foi de $23 \%$; em $3 \%$ esta informação foi ignorada.

Dados do Sistema de Informação sobre Nascidos Vivos (SINASC) mostram que, em $47 \%$ dos casos de crianças que nascem vivas no Brasil, as mães tiveram sete ou mais consultas de pré-natal, variando em entre 18\%, no Amapá, e 64\%, em São Paulo e Paraná, o que leva a se questionar a qualidade desses serviços no país ${ }^{(11)}$.

Neste estudo, as mortes referentes aos quatro anos analisados foram, na sua maioria, mortes classificadas como evitáveis em $80,6 \%$ dos casos, e ocorreram devido a causas diretas em 45,16\%. Dentre as causas diretas, as complicações obstétricas que mais levaram mulheres a óbito foram as relacionadas às doenças do aparelho circulatório, complicando a gravidez, o parto e puerpério; as embolias obstétricas; e as doenças renais, complicando o período gestacional (infecções do trato urinário e pielonefrites).

No Brasil, as causas dos óbitos maternos, segundo diagnósticos específicos nas principais capitais brasileiras, no primeiro semestre do ano de 2002, foram: transtornos hipertensivos em $24,9 \%$ dos casos, o que corresponde a $37 \%$ das mortes obstétricas diretas. Aborto (11,4\%); complicações do trabalho de parto e ao parto $(10,4 \%)$ e complicações do puerpério $(8,4 \%$, sendo infecções puerperais e embolia as principais), além das causas não especificadas, com 7,5\% dos óbitos ${ }^{(4)}$.

A morte materna tardia é a morte de uma mulher devido a causas obstétricas diretas ou indiretas, que ocorre num período superior a 42 e inferior a um ano após a gravidez ${ }^{(1)}$. O percentual de óbitos tardios encontrados neste estudo foi de $16,13 \%$. Os casos analisados e classificados como óbitos inevitáveis foram responsáveis por $16,2 \%$ destas mortes. E as causas obstétricas indiretas foram de $38,71 \%$.

Outra análise relevante feita nos relatórios da Secretaria Estadual de Saúde é compreender, por meio da sequência dos fatos e das informações coletadas nas entrevistas domiciliares e hospitalares pelos Comitês de Mortalidade Materna, que essas mulheres ao chegarem ao hospital de referência já haviam passado por outros serviços e sua condição no geral era sempre muito grave.

De posse dos documentos analisados verificouse que mesmo que os cuidados em unidade intensiva tenham sido realizados, essas mulheres, na verdade já estavam em uma condição de "óbito" durante a condução do seu pré-natal, ou na falta dele e até mesmo na deficiência da atenção básica, por não terem sido orientadas quanto à necessidade de uso de métodos contraceptivos no intuito de evitar uma gravidez, pois na ocorrência de uma gravidez nestas condições, suas vidas estariam em risco, por serem estas portadoras de doenças sistêmicas graves ou mesmo por fatores de risco gestacionais. Entretanto, no caso de ocorrência de gestação, estas mulheres sendo acompanhadas por um serviço de referência de alto risco, teriam uma melhor condição se adequadamente acompanhadas e tratadas durante o pré-natal, parto e puerpério

Neste estudo os óbitos maternos ocorreram com maior frequência entre mulheres brancas (74\%) seguido pelo grupo das negras (16\%) e por último, as pardas $(10 \%)$ e nenhum óbito de mulheres indígenas e amarelas foi registrado.

A variável raça/cor deve ser analisada com 
especial atenção, pois permitem avaliar questões sociais e, até mesmo, de acesso aos serviços, pois a ocorrência dos óbitos maternos em sua maioria, acontecem nas classes sociais mais baixas, com baixos salários e escolaridade ${ }^{(12)}$.

\section{CONSIDERAÇÕES FINAIS}

O período gestacional, entendido como um fenômeno fisiológico e que deve estar livre de maiores intercorrências, é afetado quando ocorrem complicações, e todo o ambiente de alegria e satisfação transforma-se em ansiedade, medo e tensão, já que mãe e filho estão ameaçados, bem como o restante da estrutura familiar. Além disso, o próprio sistema de saúde fracassa quando a qualidade da assistência não é satisfatória, uma vez que recursos materiais, estruturais e humanos não atendem à sua função, que é preservar a vida.

Os índices de mortalidade e o perfil das mulheres que morreram no período gestacional ou puerperal, neste estudo, não diferem de outros estudos realizados em capitais do nosso país ${ }^{(14)}$. Comparado com as taxas de outros países em desenvolvimento, nos quais menos de $62 \%$ das mulheres recebem assistência de algum tipo de profissional especializado - segundo a Organização Mundial da Saúde ${ }^{(15)}$ - , representamos uma situação de maior "estabilidade". Entretanto, ainda há muito a ser feito e melhorado, principalmente na atenção básica, no que se refere ao planejamento familiar e diagnóstico precoce das patologias gestacionais e do puerpério.

É importante ressaltar as limitações que o Comitê Hospitalar de Investigação de Mortes Maternas encontra ao acessar os prontuários para obter informações relacionadas à assistência hospitalar prestada às mulheres. Pois, mesmo que os dados referentes às variáveis sociodemográficas façam parte dos formulários, observam-se lacunas em seu preenchimento, talvez pelo fato dos profissionais responsáveis considerarem as informações desnecessárias ou por desconhecerem a utilidade dessas informações para o sistema de saúde.

Nesta pesquisa a maior dificuldade na precisão das informações foi em relação aos dados sociodemográficos, principalmente a renda, com $32 \%$ de fichas com campos não preenchidos e o número de residentes no domicílio, com $25 \%$ de campos em branco. Também ocorreu em $25 \%$ dos casos a omissão de informações sobre o número de consultas realizadas no pré-natal.
Os profissionais da área da saúde ainda precisam melhorar a qualidade das informações registradas nos prontuários de seus pacientes. Sem elas, estudos são inviabilizados e a fidedignidade da informação se perde quando não se consegue compilar um número significativo de dados e, assim, dificultase o conhecimento da realidade. Os dados permitem oportunizar a criação de estratégias e ações de saúde mais contundentes e que efetivamente vão ao encontro dass necessidades da população.

É necessário sempre retornar à discussão da ampliação do acesso e da qualidade e das políticas públicas de saúde voltadas à mulher, pois as ações governamentais são fundamentais para incentivar e apoiar os profissionais a repensar a assistência prestada. Além do mais, também é necessário capacitálos e sensibilizá-los constantemente, de forma que o atendimento prestado seja satisfatório e resolutivo.

\section{REFERÊNCIAS}

1. Ministério da Saúde (BR). Manual dos Comitês de Mortalidade Materna. Série A: normas e manuais técnicos. Secretaria de Atenção à saúde. Departamento de ações programáticas estratégicas. Brasília; 2007.

2. World Health Organization. Managing eclampsia. Educational Material for teachers of midwifery. Department of Making Pregnancy Safer. Family and Community Health. Geneva; 2006

3. Ministério da Saúde (BR). Uma análise da situação de saúde. Secretaria de Vigilância em Saúde. Departamento de Análise de Situação em Saúde. Brasília; 2007.

4. Ministério da Saúde (BR). Estudo da mortalidade de mulheres de 10 a 49 anos, com ênfase na mortalidade materna: relatório final. Brasília; 2006

5. Secretaria Estadual de Saúde (PR). Estudo de casos de óbitos maternos. Comitê Estadual de Prevenção da Mortalidade Materna. Secretaria Estadual da Saúde 2004 a 2007.

6. Secretaria Estadual de Saúde (PR). Governo lança programa "Nascer no Paraná Direito a Vida" em Curitiba. [Internet] [acesso em 23 jul 2009]. Disponível: http://tiny. cc/dwg08

7. Tobar F, Yalour MR. Como fazer teses em saúde pública: conselhos e idéias para formular projetos e redigir teses e informes de pesquisa. Rio de Janeiro: Fiocruz, 2001. 
8. Ministério da Saúde (BR). Gestação de alto risco. Manual Técnico. Secretaria de Políticas de Saúde. Departamento de Gestão de Políticas Estratégicas. Área técnica de saúde da mulher. Brasília; 2000.

9. Secretaria Estadual de Saúde (PR). SESA/ISEP/CIDS Centro de Informações e Diagnóstico em Saúde. [Internet] [acesso em 23 jul 2009]. Disponível: http:// tiny.cc/g7o9t

10. Secretaria Estadual de Saúde (PR). Programa Estadual de Gestação de Alto Risco. [Internet] [acesso em 10 abr 2009]. http://tiny.cc/vi2g5

11. Ministério da Saúde (BR). Estudo da mortalidade de mulheres de 10 a 49 anos, com ênfase na mortalidade materna: relatório final. Brasília; 2006.

12. Martins A. Mortalidade materna de mulheres negras no Brasil. Cad Saúde Pública. 2006; 22(11): 2473-9.

13. Secretaria Municipal de Saúde (PR). Pré-natal, parto, puerpério e atenção ao recém-nascido - Programa Mãe Curitibana. Curitiba; 2007.

14. Cecatti JG, Albuquerque R M, Ardí E, Faúndes. A mortalidade materna em Recife: causas de óbitos maternos. Rev Bras Ginecol Obstetr. 1998(1): 455-62.

15. World Health Organization. Fact sheet. Department of Making Pregnancy Safer. Department of Child and Adolescent Health and Development. Department of Gender, Women and Health. Department of Reproductive Health and Research. Switzerland; 2008. 